Ciencia y Educación, Vol. 4, No. 3, septiembre-diciembre, 2020

ISSN (impreso): 2613-8794・ISSN (en línea): 2613-8808

DOI: https://doi.org/10.22206/cyed.2020.v4i3.pp133-143

\title{
Desafíos de la Educación Especial en el desarrollo de escuelas inclusivas
}

\author{
Challenges of Special Education in development \\ of inclusive schools
}

Cristina Amiama-Espaillat ${ }^{a}$ Orcid: 0000-0002-8222-1530

Recibido: 27/02/2020 • Aprobado: 28/04/2020

Cómo citar: Amiama-Espaillat, C. (2020). Desafíos de la Educación Especial en el desarrollo de escuelas inclusivas. Ciencia y Educación, 4(3), 133-143. Doi: https://doi.org/10.22206/cyed.2020.v4i3.pp133-143

\section{Resumen}

Todos los países miembros de las Naciones Unidas, en el año 2015, adoptaron la Agenda 2030 para el Desarrollo Sostenible. El objetivo 4 consiste en garantizar una educación inclusiva, equitativa y de calidad y promover oportunidades de aprendizaje durante toda la vida para todos. En este artículo, se aborda el concepto de educación inclusiva que conlleva la equidad y la calidad y se exponen seis lecciones aprendidas en el contexto internacional sobre el desarrollo de escuelas inclusivas. Asimismo, se reflexiona en poblaciones específicas como los estudiantes con discapacidad y sus necesidades educativas, que tradicionalmente se ha abordado desde la Educación Especial. Finaliza con una propuesta de transformación de la Educación Especial acorde a los principios de la Educación Inclusiva.

Palabras clave: educación inclusiva; educación especial; práctica inclusiva; escuelas especiales.

\begin{abstract}
All member countries of the United Nations, in 2015, adopted the 2030 Agenda for Sustainable Development. Goal 4 is to ensure inclusive, equitable and quality education and promote lifelong learning opportunities for all. In this article, the concept of inclusive education that involves equity and quality is addressed and 6 lessons learned in the international context on the development of inclusive schools are presented. Likewise, it reflects on specific populations such as students with disabilities and their educational needs, which has traditionally been addressed since Special Education. It ends with a proposal for the transformation of Special Education according to the principles of Inclusive Education.
\end{abstract}

Keywords: Inclusive education; special education; inclusive practice; special schools

\footnotetext{
a Pontificia Universidad Católica Madre y Maestra (PUCMM), República Dominicana

Correo-e: cm.amiama@ce.pucmm.edu.do
} 


\section{Introducción}

La Agenda 2030 de las Naciones Unidas logró un gran consenso al establecer la educación inclusiva como uno de los objetivos para el Desarrollo Sostenible de las personas y del planeta; sin embargo, no necesariamente se ha traducido en políticas, culturas y prácticas en muchos países de la Región de América Latina, entre ellos la República Dominicana.

La educación inclusiva genera enormes desafíos, el primer es comprender su polisemia. En muchos países, la educación inclusiva se introdujo ligada solo a la transformación de la Educación Especial.

La educación especial ha estado buscando su sentido desde que inició el movimiento de inclusión, algunos abogan por su completa desaparición, mientras que otros les asignan nuevas funciones. En ocasiones, en esos debates se confunde su identidad con su ámbito tradicional de actuación, las escuelas especiales o con la población específica que atiende.

Este artículo aporta una revisión bibliográfica en dos sentidos: la educación inclusiva y algunas lecciones ya aprendidas en los sistemas educativos para poder cumplir con el compromiso asumido de una educación de calidad con equidad, lo que implica que todos los niños, niñas y jóvenes aprendan y participen juntos en la escuela de su comunidad. Esta última afirmación transforma no solo los servicios educativos que tradicionalmente aporta la educación especial, sino las concepciones mismas de diferencia versus normalidad, en la que se sustentaba esta disciplina. Lo que implica, un nuevo planteamiento epistemológico sobre las diferencias individuales basada en su identidad como disciplina.

\section{La educación inclusiva}

El periplo que ha transitado la educación inclusiva no ha sido único, ni lineal, ni biunívoco en sus referentes pedagógicos (Parillas, 2002); en muchas ocasiones se utiliza como sinónimo del Movimiento de Integración que se originó en los países europeos como consecuencia de la lucha de las personas con discapacidad por el derecho a participar con igualdad de oportunidades y condiciones en la sociedad (Blanco, 1999), por lo que se promovió y legisló que los estudiantes de los centros de educación especial se escolarizarán en centros educativos regulares.

Desde la perspectiva epistemológica, la educación inclusiva se puede concebir desde distintos ámbitos: político, social, educativo; dentro de lo educativo, diferentes niveles como filosofía, modelo, principio, cultura y práctica. Ocampo (2019) se refiere a ella como una "teoría sin disciplina" donde convergen una variedad de discursos, métodos, objetos, disciplinas, compromisos éticos...También, las Naciones Unidas considera a la educación inclusiva como un Derecho Inalienable para las personas con discapacidad (Convención sobre los Derechos de las Personas con Discapacidad, 2006, art. 24).

La Convención fue ratificada en la República Dominicana en el año 2009. Esta nueva perspectiva de considerar la educación inclusiva como un derecho tiene implicaciones considerables, pues ya no se trata de principios bien intencionados, sino de un aspecto relevante que no puede obviarse en las normativas, pues se consideran actos, directos o indirectos, de discriminación (Echeita, 2017).

La Declaración Mundial sobre Educación para Todos "Satisfacción de las Necesidades Básicas de Aprendizaje" promulgada en Jomtien, en 1990, se considera el venero del movimiento de Educación Inclusiva. En esta Conferencia organizada por la UNESCO, participaron más de 155 países y 150 organizaciones acordando, entre otros aspectos la "satisfacción de las necesidades básicas de aprendizaje” (art. 1) "universalizar el acceso a la educación y fomentar la equidad", (art.3) (Unesco, 1990, pp. 3-5). Al posicionar la educación como un derecho humano fundamental, se enfatizó en grupos que tradicionalmente habían estado vulnerados de ese derecho, como los estudiantes con discapacidad $\mathrm{u}$ otras condiciones.

Cuatro año más tarde, se celebró la Conferencia de Salamanca (UNESCO \& MEC, 1994), que marcó un antes y después en la historia de la educación especial, donde participaron 92 países incluyendo República Dominicana y 25 Organizaciones. En la Declaración final, se asumen los principios de la 
Educación para todos y la creación de sistemas educativos que acojan a todos los estudiantes incluyendo a aquellos con necesidades educativas especiales. Es decir, que se reconoce que no solo los estudiantes con discapacidad son excluidos del Derecho a la Educación.

Centra la atención en las escuelas regulares como el medio más eficaz para eliminar las actitudes de discriminación, crear comunidades que acogen y construir una sociedad inclusiva (UNESCO \& MEC, 1994).

La Declaración de Salamanca aportó:

una visión de la educación muy diferente a la que, en algunos casos, tal vez en demasiados, orienta los objetivos de las reformas educativas de los gobiernos. Preocupados por las exigencias educativas de la sociedad del conocimiento y presionados para mejorar el rendimiento escolar de los alumnos, han preferido un modelo liberal y competitivo para elevar la calidad de la enseñanza (Marchesi, 2004, p. 36).

La educación inclusiva implica un cambio de mirada no solo del sistema educativo, también de la sociedad. No es una innovación educativa, no es un movimiento que pertenece a un determinado colectivo, "sino una cuestión de respeto de los derechos que afecta prioritariamente a las orientaciones de política general de un país" (Unesco, 2008, p. 6). Es la única manera de eliminar la exclusión que padecen tantos ciudadanos del mundo.

La educación inclusiva, utilizando la metáfora de Gabriel García Márquez para referirse a los excluidos, es la segunda oportunidad que tienen las estirpes condenadas a cien años de soledad (García-Márquez, 1982). La Organización para el Desarrollo la Organización para la Cooperación y el Desarrollo Económico propone que estas "segundas oportunidades" deben incluir "programas de alfabetización, cursos en el trabajo, planes para reconocer el aprendizaje informal" (OCDE, 2007, p. 5) y sistemas de apoyo a los aprendizajes que contemplen a las fami- lias que están en situación de vulnerabilidad, como el caso de los las migrantes o familias que sobreviven con escasos recursos económicos.

La educación inclusiva reconoce que la diversidad funcional es una parte más de la realidad humana; la OCDE la propone como una respuesta para mejorar la equidad en los sistemas educativos (Hernández \& Ainscow, 2018). "Un mayor nivel de equidad implica avanzar hacia la creación de escuelas que eduquen en la diversidad y que entiendan ésta como una fuente de enriquecimiento y de mejora de la calidad educativa” (Blanco, 1999, p. 56).

El Ministerio de Educación de la República Dominicana asumió la definición de la UNESCO de Educación Inclusiva: "como un proceso orientado a responder a la diversidad de necesidades de todos los estudiantes, incrementando su participación en el aprendizaje, la cultura y las comunidades, reduciendo y eliminando la exclusión en y desde la educación” (Ministerio de Educacion de la República Dominicana, 2017, p. 13). Lo cual pone énfasis en la transformación del sistema educativo completo para que todos los estudiantes, sin importar su condición, puedan acceder, participar, aprender y permanecer en los centros educativos de su comunidad.

Comprender que la educación inclusiva no está ligada a grupos específicos que tradicionalmente han estado vulnerados de su derecho a la educación, ni a su lucha por la inclusión plena en la sociedad, implica el desarrollo de nuevas políticas que construyen una nueva cultura. Asimismo, se supera la forma de atender las diferencias entre los estudiantes heredada de la escuela de la época industrial y, por tanto, se introducen nuevos modelos pedagógicos que promueven el aprendizaje de todos asumiendo y disfrutando la diversidad como condición inherente a la sociedad.

\section{Lecciones aprendidas sobre la Educación Inclusiva}

En la actualidad, casi todos los sistemas educativos han integrado el concepto de educación inclusiva en sus legislaciones, algunos con una perspectiva 
más amplia, otros todavía restringida a grupos específicos, como los estudiantes con discapacidad. En ese sentido, la educación inclusiva tiene fundamento desde tres perspectivas como proyecto contra el fracaso escolar y la exclusión; como un cambio cultural en la escolarización actual y futura, y como un compromiso político para un mundo más justo (Azorín, Arnaiz, \& Maquilón, 2017, p. 1022).

Casi todos los sistemas educativos, en su trayecto para la educación de estudiantes con dificultades o con otros tipos de exclusión como de género, étnico, social, han transitado de forma gradual de la exclusión a la inclusión, con dos fases intermedias denominadas segregación e integración (Ainscow, 2012; Blanco, 1999; Hernández \& Ainscow, 2018; Parillas, 2002). Como ejemplo, la historia de educación de las mujeres o de afroamericanos en Estados Unidos.

El tránsito a la educación inclusiva ha dejado muchas lecciones aprendidas. Mel Ainscow, uno de los investigadores con más prestigio en este ámbito, comparte seis lecciones sobre la implementación de la educación inclusiva en los Sistemas Educativos Internacionales (Ainscow, 2012). (Para conocer el desarrollo de la educación inclusiva en América Latina y el Caribe véase Blanco, 2011; Payá, 2010).

\section{Lección 1: para seguir avanzando en las políticas y las prácticas inclusivas, es necesario definir qué es la educación inclusiva.}

Ainscow propone unas series de principios que deben regir en la educación inclusiva y a su vez propone cuatro características básicas que deben estar presentes en su definición : a) la educación inclusiva implica la atención de todos los niños y jóvenes; b) además del acceso y la presencia de los estudiantes en los centros escolares, se propicia la participación y se aseguran los aprendizajes definidos en el currículo; c) supone una lucha activa contra la exclusión de grupos específicos, y d) la inclusión es un proceso, por tanto ningún centro educativo ha alcanzado el estado de perfección y necesita una evaluación continua (Ainscow, 2012).
Lección 2: las prácticas inclusivas suponen vencer las barreras a la participación y el aprendizaje e implican la reorganización de los recursos humanos disponibles.

Florian y Black-Hawkins (2011) realizaron un estudio en 11 escuelas de Escocia para identificar buenas prácticas inclusivas. Determinaron que lo más importante es la forma (fundada en sus creencias) en que los docentes responden a las diferencias en el aula, asimismo que no hay diferencias significativas entre las estrategias de enseñanza que usan los docentes de educación especial y las utilizadas por los docentes de las escuelas regulares.

Ainscow (2012) afirma que se debe explorar otras posibilidades para la eliminación de barreras en el aula que no provengan de la transferencia de las estrategias de la educación especial. En ese sentido, existen sólidas propuestas como la Enseñanza Diferenciada (Tomlinson, 2013, 2017) y el Diseño Universal de los Aprendizajes (Alba, 2016) las cuales están relacionadas con el compromiso de personalizar la enseñanza y con los aprendizajes de todos los estudiantes.

Los sistemas de apoyo a estudiantes con necesidades educativas específicas que se basan en realizar ajustes curriculares individualizados y en tener profesores de apoyo (sombra) solo exclusivo para trabajar con determinados niños en el aula son ejemplos de esas transferencias de la educación especial a la regular.

Otra lección aprendida se relaciona con los recursos humanos disponibles. El apoyo entre pares es una de las medidas más eficaces para mejorar los aprendizajes, por tanto, en clases con muchos estudiantes por aulas, se disponen de más recursos humanos para la atención a la diversidad.

\section{Lección 3: motivar a los docentes a desarrollar prácticas más inclusivas a partir de las investiga- ciones disponibles}

El desarrollo de escuelas inclusivas debe basarse en evidencias que conduzcan a la reflexión. Esto supone romper con concepciones elaboradas desde la 
experiencia y de las creencias culturales, incluso de mitos, para construir con base en investigaciones con aval científico.

Algunos investigadores han indagado sobre formas "más justas y potenciadoras" para comprender la diversidad de los alumnos, que no sea basada en sus capacidades (Ainscow, 2012) y sostienen que muchos de los conocimientos disponibles sobre la enseñanza coadyuvan al desarrollo de una práctica inclusiva en el aula, es decir, "puede ayudar a crear un espacio para reexaminar y replantear, por medio de la ruptura de los discursos vigentes y concentrando nuestra mayor atención en las posibilidades que han sido pasadas por alto de hacer avanzar la práctica (p. 5).

Un principio que mueve a los docentes a desarrollar práctica inclusiva es la convicción de que el rendimiento académico de los estudiantes, sin importar su condición, mejora si se les ofrecen los recursos y apoyos adecuados.

El cambio de práctica de los docentes solo es posible mediante la reflexión, entendida como una acción de "analizar nuestra experiencia a la luz de las evidencias recogidas y, después, valorarlas mediante el contraste pertinentes; la experiencia de otros, los avances del conocimiento, la literatura especializada, etc." (Domingo \& Gómez, 2017, p. 10). Otra herramienta eficaz para el desarrollo de las escuelas inclusivas y para promover la reflexión en la práctica es la observación recíproca.

Lección 4: el apoyo pedagógico a los alumnos requiere de una planificación meticulosa y de formación adecuada de todos los actores

En la educación inclusiva existen estudiantes que necesitan apoyos y recursos adicionales a los habituales para acceder y participar de los aprendizajes. Todos los sistemas de apoyo presentan ventajas y desventajas. Takala et al., (citados en Ainscow, 2012) describieron el uso de tres enfoques empleados en Finlandia que se basan en paradigmas diferentes, el último redefine el rol de los docentes de Educación Especial en un entorno inclusivo. a. Aulas especiales en centros educativos regulares, es decir, que un docente de Educación Especial atiende, en un espacio diferenciado de los niños de su edad, a estudiantes con necesidades especiales, en muchas ocasiones, con discapacidad. Es una medida que tienden a producir mayor estigmatización a este grupo de estudiantes y a no propiciar la atención a la diversidad en el conjunto del centro educativo.

b. Enseñanza de grupos reducidos. Consiste en ofrecer apoyo a un grupo de estudiantes fuera del aula con otro docente en determinadas asignaturas. Las dificultades que plantea este modelo, además de la estigmatización de los estudiantes, son la falta de coordinación entre los docentes y la pérdida de informaciones relevantes por parte de los estudiantes por estar fuera del salón de clases.

c. Dos docentes en la misma aula (Enseñanza colaborativa), en ella el docente de educación especial comparte el escenario en el aula y ofrece el apoyo en el mismo contexto, no solo a los estudiantes con necesidades específicas de apoyo educativo, sino que es un colaborador del docente para implementar estrategias que favorecen la atención a la diversidad.

Para avanzar en la educación inclusiva se hace necesario una reflexión sobre el profesor de apoyo dentro del aula ("profesor sombra"). Diferentes estudios demuestran que en muchas ocasiones se convierte en un sustituto del docente y su rol es ofrecer clases particulares dentro del gran grupo con intencionalidad pedagógica diferente, lo cual promueve una segregación dentro del aula.

Para transitar del modelo de integración a inclusión, es necesario que el docente asuma el compromiso de los aprendizajes de todos sus estudiantes, incluyendo a aquellos que reciben apoyo, desde su planificación y práctica pedagógica asegurando la participación de todos; esto implica un cambio en la organización y metodología del aula de centrada 
en la transmisión de saberes a la construcción colaborativa y el desarrollo de competencias (Soldevila, Naranjo, \& Muntaner, 2017).

Por otro lado, los profesores de apoyo deben recibir un entrenamiento específico que contemple el modo y la organización del aula (Ainscow, 2012; Ainscow, Hopkins, Southworth, \& West, 2001) para facilitar su cambio de paradigma, del rehabilitador, centrado en el nińo y "sus necesidades", al educativo, en colaboración y apoyo al docente en la implementación de estrategias activas y colaborativas, como los proyectos de aula, los cuales permiten eliminar las barreras para la participación y el aprendizaje. Basado en lo anterior, el Ministerio de Educación de la República Dominicana (2017) recomienda incluir el Diseño Universal de los Aprendizajes (DUA) en el salón de clases, antes de realizar Ajustes Curriculares Individualizados (ACI) e incluirlas en la planificación del aula.

Lección 5: en todas las escuelas inclusivas se valora de forma positiva la diversidad, sin importa la forma en que se organicen

Las escuelas inclusivas tienen unas características que las diferencias del resto: la forma de acoger a todos los estudiantes, el clima afectivo y emocional en la escuela y en el aula (Blanco, 1999, p. 70). El modo de organización propuesto en las escuelas eficaces ha demostrado ser muy adecuado para las escuelas inclusivas (Ainscow et al., 2001).

La cultura organizativa afecta a toda la comunidad educativa y no solo a lo que sucede en el aula. En esta perspectiva, los estudiantes que tienen dificultades para adaptarse a las normas establecidas por el Centro Educativo no se consideran como "casos problemáticos", sino que son oportunidades de mejora y de desafío que generan una reflexión de las prácticas existentes.

Una herramienta muy poderosa para el desarrollo de cultura inclusiva es el índex de Inclusión, que integra todos los enfoques para la mejora escolar. Propone que la educación inclusiva se fundamente en tres pilares: políticas, prácticas y culturas, esta última "refleja las relaciones los valores y las creencias profundamente arraigadas en la comunidad educativa" (Ainscow \& Booth, 2015, p. 17), sin cambio de cultura no es posible sustentar la mejora. La cultura escolar inclusiva se establece construyendo comunidad y estableciendo los valores inclusivos.

\section{Lección 6: los líderes escolares tienen un papel principal en el fomento de una cultura escolar inclusiva}

Ainscow (2012) referencia la investigación de Zollers y colaboradores en 1,000 centros educativos estadounidenses que concluyó en identificar siete elementos comunes en las prácticas escolares inclusivas efectivas, el primero fue el liderazgo visionario, hallazgo corroborados por otras investigaciones sobre el tema como la de Chadbourne en 1997 y la de Kugelmass y Ainscow de 2004.

Para fomentar el desarrollo de escuelas inclusivas es imprescindible un liderazgo compartido, centrado en el aprendizaje. Para ello el director debe centrarse en la dimensión pedagógica colocando lo administrativo al servicio de ésta. Los líderes eficaces hacen que la organización funcione bien, pues crean comunidades horizontales que transforman la cultura individualista en colaborativa (Bolívar, López, \& Murillo, 2013)

Basado en Riehl concluye que los líderes educativos deben focalizarse en tres tareas esenciales: fomentar nuevos significados sobre la diversidad, promover prácticas inclusivas y desarrollar una relación entre la escuela y la comunidad.(Para ampliar sobre el liderazgo compartido en las organizaciones escolares véase Bolivar, 2000).

\section{Educación Especial}

La Educación Especial puede ser analizada desde la perspectiva de disciplina científica o como modalidad educativa. En tanto disciplina ha evolucionado en la conceptualización de su objeto de estudio. Al inicio y desde una perspectiva médica-rehabilitadora se centraba en el déficit de los individuos y 
buscaba diferentes maneras para "compensarlo" — se desarrollaron el Sistema Braille, la Lengua de Señas y métodos específicos para la enseñanza como el de María Montessori o el de Ovide Decroly_; en los años noventa, con el movimiento de integración, el énfasis se trasladó a los apoyos y recursos necesarios para su participación plena en la sociedad y actualmente, se le considera como sujetos de derecho.

Esta transformación de la comprensión del objeto de la Educación Especial está influenciada por los cambios de las diferentes disciplinas: la psicología (educativa, del aprendizaje y social), la pedagógica, la neurociencia, la sociología, la antropología, la medicina, la política. Hoy en día, no se pone en duda que las dificultades no están en las personas, sino en la interacción con las barreras que encuentran en la sociedad que les limita su acceso, participación y aprendizaje en igualdad de oportunidades.

Como modalidad educativa, la historia de escolarización de estudiantes con discapacidad (véase una breve historia de la Educación Especial en el República Dominicana en Amiama-Espaillat, 2016) ha estado marcada por la exclusión, segregación y la lucha por la igualdad de oportunidades en un entorno inclusivo. Hasta el informe Warnock (1978), La educación de estudiantes con discapacidad se entendía como exclusivamente competencia de la educación especial y restringida a esa modalidad educativa.

A finales del siglo xIX, los países comenzaron a ofrecer la educación como un bien público para todos sus ciudadanos, sin importar su condición. Esta excelente decisión conllevó un considerable aumento en la cantidad de niños que asisten a instituciones educativas, se organizaron los sistemas nacionales de escolarización.

Paralelamente, la psicología se separa de la filosofía e inicia el auge de las pruebas psicométricas. La primera prueba de inteligencia de Binet y Simon surge a petición del Ministro de Educación de Francia con el fin de poder distinguir a los niños que estaban presentando retrasos significativos en los aprendizajes, surgen las primeras escuelas y clases especiales.

Los centros de Educación Especial, como modalidad paralela, nacen con la buena intención de ofrecer una educación especializada a una población de niños y niñas que tenían dificultades con los aprendizajes, ya se habían desarrollado métodos específicos para poblaciones específicas como el sistema Braille para los estudiantes ciegos (en 1829 se creó la primera escuela de ciegos en Estados Unidos).

Con el informe Warnock (1978), aunque estaba limitado a la educación especial en Inglaterra, se pone en relevancia que esta modalidad educativa limitaba las oportunidades de aprendizaje y participación de los estudiantes con discapacidad (en ese momento, se denominaba "deficientes") por estar fundamentado en un paradigma médico y basarse en programas individualizados rehabilitadores para "compensar" lo que se catalogaba como "déficit".

$\mathrm{El}$ informe introduce un nuevo paradigma para la educación de estudiantes con discapacidad. Inicia reconociendo el derecho a la educación de todos los niños y estableciendo los mismos fines para todos. En la República Dominicana, estos dos principios se introdujeron en la Ley de Educación 67-97 y sus respectivas Ordenanzas (1'95 que establecía el Currículo y 1'96, la evaluación) a partir de los cuales, el currículo que rige a los centros educativos especiales es el mismo para todos. Con ello, se eliminaron los programas de desarrollo individuales que se basaban en la "rehabilitación" de la deficiencia.

Otro de los principios del Informe Warnock, que supuso una ruptura con el paradigma imperante, es que las necesidades educativas son comunes a todos los niños, y, por tanto, no deben existir dos grupos, aquellos considerados "deficientes" que deben asistir a educación especial y los "normales" que reciben simplemente educación.

Por otro lado, se establece una línea continua en los servicios que se ofrecen en la educación especial, desde la ayuda temporal hasta las adaptaciones permanentes al currículo. A la vez, se establece que, sin importar el lugar donde se ofrezcan, dichos servicios deben tener un carácter suplementario, nunca paralelo. Por último, puntualiza que el sistema de categoría no debe basarse en las necesidades específicas de los individuos, sino en los apoyos y recursos 
requeridos, aspecto que es uno de los menos desarrollados a lo largo de estas décadas.

Este informe es considerado un hito en la educación de estudiantes con discapacidad, ya que el movimiento de integración educativa cobra un oportuno impulso: los niños con discapacidad acceden al currículo general, se amplía el ámbito de la Educación Especial al introducir el término de Necesidades Educativas Especiales (NEE) y al definir los apoyos necesarios para que los niños accedan, aprendan y participen de la educación de todos, en los centros regulares.

En la actualidad, coexisten varias opciones para la escolarización de estudiantes con discapacidad, desde entornos inclusivos, sin apoyo, a entornos restringidos como los centros de educación especial; con el intermedio de aulas específicas de educación especial en centros regulares. Se han diversificado las modalidades de participación de estudiantes de centros de educación especial en las escuelas regulares.

Lo anterior evidencia, que aún en la construcción colectiva se consideran "diferencia" y "déficit" como sinónimo; por tanto, se deben proveer apoyos para "compensar" el déficit, pero en nuevos entornos. La educación inclusiva asume una epistemología diferente sobre el ser humano y sus "diferencias" y, por tanto, propone una transformación a las instituciones educativas para que tengan los recursos de educar a todos sin importar sus condiciones, se trata de comprender, en palabras de Edgar Morín "la unidad dentro de lo diverso, lo diverso dentro de la unidad, reconocer, por ejemplo, la unidad humana a través de las diversidades individuales y culturales, las diversidades individuales y culturales a través de la unidad humana” (citado en Boggino, 2011, p. 61).

\section{Transformación de la Educación Especial}

Cigman (2007) reflexiona en torno a dos tipos de principios, que en ocasiones generan fuertes conflictos, que rigen el desarrollo de escuelas inclusivas, los denomina principios universalistas y moderados; la diferencia fundamental entre estos dos grupos son las Escuelas de Educación Especial.
Mientras los universalistas afirman que los centros de educación especial son segregadores en sí mismos porque favorecen y eternizan la discriminación, los moderados, por su parte, no consideran incompatible la existencia de centros de educación especial para un grupo muy minoritario de niños que presentan condiciones específicas en su desarrollo y que las escuelas llamadas regulares no disponen de los recursos necesarios para asegurarles una educación integral basada en sus necesidades básicas.

Casi todos los países que han avanzado en la inclusión educativa mantienen una atención paralela a los estudiantes con discapacidad severa, pero con diferencias significativas con relación a las escuelas de principio de siglo xx: están organizadas con base en los currículos nacionales con el fin de ofrecer una respuesta educativa que permita el desarrollo de las capacidades de sus estudiantes hasta donde sus aptitudes los limiten. Por otro lado, han desarrollado programas innovadores que vincula la comunidad con la escuela.

Han pasado 25 años de la Declaración de Salamanca y todavía hoy las acciones propuestas tienen una gran relevancia para los sistemas educativos, y especialmente, para el dominicano. Echeita y Verdugo (2004) plantean que ante la compleja realidad educativa se necesitan todas las luces posibles para entender y actuar y que esta Declaración puede guiar el pensamiento y las acciones de todos los involucrados en las tareas educativas.

Se reconoce un proceso de avance en todos los países de América Latina y el Caribe en el desarrollo de escuelas inclusivas, pero aún persisten muchos desafíos, posiblemente como lo expresa Cruz (2019), porque se ha buscado soluciones simples a problemas complejos.

Pilar Arnaiz, en su reflexión sobre la educación inclusiva en el siglo XxI, plantea algunos pendientes: a) los cambios radicales que se deben producir a nivel organizativo, curricular y metodológico en los sistemas educativos que deben concretizarse en el aula. b) Generalizar la educación inclusiva como una realidad comunitaria, institucional y personal, más allá de una experiencia o para algunos casos. 
c) La formación inicial y permanente de los docentes desde el compromiso ético que implica una renovación pedagógica auténtica que permee a todos los niveles del sistema educativo (incluye a los poderes públicos, a los administradores...), y por último, d) superar la dispersión de medidas específicas para grupos vulnerados e iniciar con proyectos inclusivos que contemple a todos (Arnaiz, 2019).

La Ley de Educación 67-97 (en proceso de revisión) concibe la Educación Especial en el país como "un Subsistema que tiene como objeto atender con niveles de especialización requerida a los niños y jóvenes que poseen discapacidades o características excepcionales" (Art. 48 y 49).

Es fundamental, en los niveles de análisis y toma de decisión política, no utilizar como sinónimo educación especial y escuelas especiales. La educación especial debe reconvertirse en un sistema transversal que ofrezca los recursos y apoyos educativos en todos los niveles. Implica ampliar la mirada para identificar a todos los estudiantes con barreras para acceder, permanecer y aprender en la escuela de su comunidad.

Hasta tanto no logremos el objetivo del desarrollo sostenible 4, Garantizar una educación inclusiva, equitativa y de calidad y promover oportunidades de aprendizaje durante toda la vida para todos, los centros de Educación Especial, y algunas medidas para la atención a la diversidad en los centros educativos regulares (Ministerio de Educación de la República Dominicana, 2017), constituyen una forma de acceso, de aprendizaje y de participación con muchos efectos secundarios.

Las escuelas de educación especial deben continuar, como lo establece la mayoría de normativa de los países de América Latina, ofreciendo atención diferenciada basada en el currículo escolar a estudiantes gravemente afectados para brindar oportunidades continuas para su inclusión social y laboral y abrir sus puertas a la comunidad.

El Art. 24 de la Convención sobre los Derechos de las Personas con Discapacidad compromete a los Estados Miembros a ofrecer una educación inclusiva en todos los niveles; pero a la vez establece que deben recibir el apoyo requerido para que puedan participar en igualdad de oportunidades; sin proponérselo plantean el dilema de la diferencia descrito por Martha Minow, a la vez que generan interrogantes como las propuestas por Florián (2013) “¡cómo puede proporcionarse apoyo especializado sin mantener la segregación y la discriminación? ¿Hay futuro para la educación especial en la respuesta a esta pregunta?” (p.29).

Torres (2011) plantea que la identidad científica de la educación especial es la que debe marcar el sendero de cambio en la educación inclusiva. Se hace necesario asumir un compromiso con nuevas formas de apoyo que potencien las capacidades de los seres humanos en las instituciones educativas que acoge a todos los estudiantes, lo que implica superar el lenguaje de las diferencias, además, de implementar estrategias para que todos participen y aprendan más allá de sus condiciones personales de desarrollo, focalizadas no en los individuos, sino en la eliminación de las barreras que enfrentan en las instituciones educativas y sociales para participar y aprender.

\section{Referencias}

Ainscow, M. (2012). Haciendo que las escuelas sean más inclusivas: lecciones a partir del análisis de la investigación internacional. Revista de Educación Inclusiva, 5(1), 39-49.

Ainscow, M., \& Booth, T. (2015). Guía para la Educación Inclusiva Desarrollando el aprendizaje y la participación en los centros escolares. OEI, FUHEM. Recuperado a partir de https:// dds.cepal.org/redesoc/publicacion?id $=4160$

Ainscow, M., Hopkins, D., Southworth, G., \& West, M. (2001). Hacia escuelas eficaces para todos: manual para la formación de equipos docentes. Madrid: Narcea.

Alba, C. (2016). Diseño Universal para el Aprendizaje: Educación para todos y prácticas de enseñanza inclusivas. Madrid: Morata.

Amiama-Espaillat, C. (2016). Respuesta educativa para la atención de estudiantes con Trastorno del Espectro Autista. Santo Domingo: Organización de Estados Iberoamericanos (OEI). 
Arnaiz, P. (2019). La educación inclusiva en el Siglo XXI. Avances y desafíos. Universidad de Murcia, 53(9), 1689-1699. doi:10.1017/ CBO9781107415324.004

Azorín, C., Arnaiz, P., \& Maquilón, J. (2017). Revisión de Instrumentos sobre Atención a la Diversidad para una Educación Inclusiva de Calidad. Revista Mexicana de Investigación Educativa, 22(75), 1021-1045.

Blanco, R. (1999). Hacia una escuela para todos y con todos. Boletin del Proyecto Principal de Educación para América Latina y el Caribe, (48), 55-72. Recuperado a partir de http:// www.oei.es/n2909.htm

Blanco, R. (2011). Educación inclusiva en América Latina y el Caribe. CEE Participación Educativa, 18(46-59), 2-3.

Boggino, N. (2011). Los problemas de aprendizaje no existen. Propuestas alternativas desde el pensamiento de la complejidad (Vol. 1). Rosario: HomoSapiens.

Bolivar, A. (2000). El liderazgo compartido según Peter Senge. En III Congreso Internacional sobre Dirección de Centros Educativos (pp. 459-471). Bilbao: ICE de la Universidad de Deusto.

Bolívar, A., López, J., \& Murillo, F. J. (2013). Liderazgo en las Instituciones Educativas. una Revisión de Líneas De Investigación. Revista Fuentes, 14, 15-60.

Cigman, R. (2007). A question of universality: Inclusive education and the principle of respect. Journal of Philosophy of Education, 41(4), 775-793. Doi:10.1111/j.14679752.2007.00577.x

Cruz, R. (2019). A 25 Años de la Declaración de Salamanca y la Educación Inclusiva: Una Mirada desde su Complejidad. Revista latinoamericana de educación inclusiva, 13(2), 75-90. Doi: $10.4067 / s 0718-73782019000200075$

Domingo, Á., \& Gómez, M. V. (2017). La práctica reflexiva. Bases, modelos e instrumentos. Madrid: Narcea, S.A. De Ediciones.

Echeita, G. (2017). Educación inclusiva. Sonrisas y lágrimas. Aula Abierta, 46, 17. Doi:10.17811/ rifie.46.2017.17-24
Echeita, G., \& Verdugo, M. Á. (2004). La Declaración de Salamanca sobre Necesidades Educativas Especiales diez años después: Enseñanza y aprendizaje de un evento singular. En La Declaración de Salamanca sobre Necesidades Educativas Especiales 10 años después. Valoración y prospectiva (pp. 11-22).

Florián, L. (2013). La Educación Especial en la era de la Inclusión: ¡el fin de la educación especial o un nuevo comienzo? Revista Latinoamericana de educación inclusiva, 7(2), 27-36.

García-Márquez, G. (1982). La Soledad de América Latina. En Discurso de aceptación del Nobel de Literatura. Recuperado a partir de http:// estaticos.elmundo.es/especiales/cultura/ gabriel-garcia-marquez/pdf/discurso_gabriel_ garcia_marquez.pdf

Hernández, M. A., \& Ainscow, M. (2018). Escuela Inclusiva Tecnológica Equidad e Inclusión: Retos y Progresos de la Escuela del siglo XXI. Retos XXI, 2, 15-22.

Marchesi, Á. (2004). Salamanca: El sentido de la educación. En La Declaración de Salamanca sobre Necesidades Educativas Especiales 10 años después. Valoración y prospectiva (pp. 35-38). Salamanca: INICIO. Recuperado a partir de http://riberdis.cedd. net/bitstream/handle/11181/3223/ Declaracion_de_Salamanca.pdf?sequence $=1 \&$ rd=003164792425720

Ministerio de Educación de la República Dominicana (2017). Orientaciones Generales para la Atención a la Diversidad Guía para la realización de Ajustes Curriculares Individualizados ( $A C I$ ). Santo Domingo, República Dominicana: Minerd. Recuperado a partir de https:// goo.gl/5jvcJX

Naciones Unidas. Convención sobre los Derechos de las Personas con Discapacidad (2006). Recuperado a partir de https://www.un.org/esa/ socdev/enable/documents/tccconvs.pdf

Ocampo, A. (2019). Educación Inclusiva: una reflexión aproximada sobre la formación del método. CEDOTIC. Revista de la Facultad de Ciencias de la Educación, 4(2), 26-51. 
OCDE. (2007). Sintesis: Diez pasos hacia la equidad en la educación. París: OCDE. Recuperado a partir de http://www.oecd.org/education/ school/40043349.pdf

Este referencia aparece en la página 6, estaba correcta, pero modifiqué la forma de referencia para mayor claridad.

Parillas, Á. (2002). Acerca del origen y sentido de la educación inclusiva. Revista de educación, 327, 11-29.

Payá, A. (2010). Políticas de educación inclusiva en América Latina. Propuestas, realidades y retos de futuro. Revista Educación Inclusiva, 3(2), 125-142.

Soldevila, J., Naranjo, M., \& Muntaner, J. J. (2017). El profesor de apoyo: del modelo de integración a la inclusión educativa. Prácticas Innovadoras inclusivas: retos y oportunidades (Vol. 53). Oviedo: Servicios de Publicaciones de la Universidad de Oviedo.

Tomlinson, C. A. (2013). El aula diversificada. Dar respuestas a las necesidades de todos los estudiantes. Barcelona: Ediciones Octaedro, S.L.

Tomlinson, C. A. (2017). How to differentiate Instruction in Academically Diverse Classrooms (3 Kindle.). Alexandria, VA USA: ASCD. Recuperado a partir de amazon.com
Torres, J. (2011). La identidad científica de la educación especial: marcando el sendero de la inclusión educativa. Revista Internacional de Investigación de Ciencias Sociales, 7(1), 137-162.

Unesco. (1990). Declaración Mundial sobre Educación Para todos y Marco de Acción para satisfacer las necesidades básicas de aprendizaje. Jomtiem.

Unesco. (2008). La Educación Inclusiva: El camino hacia el futuro". ED/BIE/CONFINTED (Vol. 48). Ginebre. Recuperado a partir de http:// www.ibe.unesco.org/fileadmin/user_upload/ Policy_Dialogue/48th_ICE/General_Presentation-48CIE-4_Spanish_.pdf

UNESCO, \& MEC. (1994). Declaración de Salamanca y Marco de Acción para las Necesidades Educativas Especiales. Recuperado a partir de https://unesdoc.unesco.org/ark:/48223/ pf0000098427_spa

Warnock, M. (1978). Special Educational Needs. London. Recuperado a partir de http:// www.educationengland.org.uk/documents/ warnock/warnock1978.html\#00 